\title{
A Hipotensão Ortostática Infrequente no Brasil: Estamos Subestimando o Problema?
}

\author{
The Uncommon Orthostatic Hypotension in Brazil: Are We Underestimating the Problem?
}

\author{
Humberto Graner Moreira ${ }^{10}$ \\ Liga de Hipertensão Arterial (LHA) - Faculdade de Medicina da Universidade Federal de Goiás, ${ }^{1}$ Goiânia, GO - Brasil \\ Minieditorial referente ao artigo: A Prevalência da Hipotensão Ortostática e a Distribuição da Variação Pressórica no Estudo Longitudinal da \\ Saúde do Adulto
}

A homeostase da pressão arterial (PA) depende de mecanismos fisiológicos complexos que envolvem interações contínuas dos sistemas cardiovascular, renal, neural e endócrino. Esses mecanismos também devem garantir a manutenção do débito cardíaco adequado, mesmo em situações de rápidas variações circulatórias. Uma dessas situações está relacionada às mudanças dinâmicas da postura, de deitado para em pé, quando a rápida redução do retorno venoso pode afetar a pré-carga, o volume sistólico e a pressão arterial média. A hipotensão ortostática $(\mathrm{HO})$ é uma manifestação de disfunção autonômica e ocorre quando os mecanismos adaptativos cardiovasculares falham em compensar essas alterações ao assumir a posição em pé. ${ }^{1}$

O diagnóstico de $\mathrm{HO}$ requer a demonstração de diminuição significativa e persistente da PA durante a ortostase, seja pelo teste de se levantar ativamente à beira leito ou pelo teste de inclinação (tilt-test). Diretrizes nacionais e internacionais endossam a definição de $\mathrm{HO}$ como uma queda $\geq 20 \mathrm{mmHg}$ na pressão arterial sistólica (PAS) ou uma queda $\geq 10 \mathrm{mmHg}$ na pressão arterial diastólica (PAD) dentro de 3 minutos após a posição ortostática, independentemente da presença de sintomas..$^{2-4}$ Essa definição foi estabelecida pela primeira vez por um consenso em 1996 e foi baseada em vários pequenos estudos fisiológicos, além de considerações pragmáticas. ${ }^{5}$ Com essa definição, evidências crescentes têm demonstrado que a $\mathrm{HO}$ prediz mortalidade por todas as causas ${ }^{6,7}$ e incidência de doenças cardiovasculares, ${ }^{7,8}$ sendo ainda mais relevante do que o descenso noturno reverso, observada na monitorização ambulatorial da PA, para predizer eventos cardiovasculares. ${ }^{9}$ Uma meta-análise recente envolvendo 121.913 indivíduos e um acompanhamento médio de 6 anos relatou que a $\mathrm{HO}$ estava associada a riscos 50, 41 e $64 \%$ maiores de morte por todas as causas, doença arterial coronariana e acidente vascular cerebral, respectivamente. ${ }^{8}$

\section{Palavras-chave}

Hipotensão Ortostática/complicações; Epidemiologia; Doenças Cardiovasculares; Acidente Vascular Cerebral; Infarto do Miocárdio; Hipertensão; Saúde do Adulto.

Correspondência: Humberto Graner Moreira •

Faculdade de Medicina da Universidade Federal de Goiás - 1a Aveninida s/n

- Setor Leste Universitário. CEP 74605-050, Goiânia, GO - Brasil

E-mail: humbertograner@uol.com.br

DOI: https://doi.org/10.36660/abc.20200352
Para determinar a prevalência de $\mathrm{HO}$ em uma população brasileira, Velten e colegas apresentam nesta edição dos Arquivos uma análise detalhada do comportamento da pressão arterial após manobras posturais em 14.833 indivíduos do estudo ELSA-Brasil. ${ }^{10}$ A coorte ELSA-Brasil incluiu 15.105 funcionários públicos de 35 a 74 anos de 5 universidades e 1 instituto de pesquisa localizados em diferentes regiões do Brasil. O estudo foi realizado de 2008 a 2010 e desenvolvido para abordar a incidência de doenças cardiovasculares e os principais fatores de risco associados entre funcionários funcionários ativos ou aposentados dessas instituições. ${ }^{11} \mathrm{~A}$ prevalência relatada de $\mathrm{HO}$ nessa população foi de 2,0\% e aumentou com a idade, chegando a 3,3\% em indivíduos entre 65 e 74 anos. Entre aqueles com triagem positiva para $\mathrm{HO}$, a presença de sintomas foi observada em $19,7 \%$, contra apenas 1,4\% entre aqueles sem $\mathrm{HO}$. Os sintomas foram relatados em até $43 \%$ dos indivíduos que apresentaram queda concomitante na PAS e PAD.

Para além de um inquérito epidemiológico, em um país onde muitos desses dados são escassos ou ausentes, este estudo levanta algumas questões que merecem ser abordadas. Primeiro, a prevalência de HO nesta coorte foi baixa. Infelizmente, não existem outros estudos na literatura que tenham investigado a prevalência de $\mathrm{HO}$ no Brasil, e esse também é outro mérito dos autores. Pesquisas epidemiológicas internacionais demonstram que a prevalência de $\mathrm{HO}$ varia de 5 a $20 \%$, mas pode chegar a 30\% em indivíduos com mais de 70 anos de idade. ${ }^{1,12}$ Neste trabalho de Velten et al., ${ }^{10}$ mesmo em indivíduos com mais de 64 anos, a prevalência ainda era muito menor do que os relatos anteriores. As razões para essa discrepância não foram claras. Uma parcela significativa dos idosos acima de 74 anos foi excluída e poderia aumentar esse número, mas as características basais do estudo ELSA-Brasil ainda apontam para uma população com alta frequência de fatores de risco: $63,1 \%$ tinham excesso de peso, $61,5 \%$ apresentavam colesterol alto, 35,8\% apresentavam PA elevada, e 20,3\% tinham tolerância à glicose comprometida. ${ }^{11}$ Se a baixa prevalência apenas reflete uma população específica, retomaremos esse tópico mais adiante neste artigo.

Segundo, como parte da avaliação do protocolo, a manobra de mudança postural incluiu medidas da PA aos 2,3 e 5 minutos após o repouso. Os autores apontam que a prevalência de $\mathrm{HO}$ pode mais que dobrar, para até 4,3\%, quando se considera a redução da PA em pelo menos uma das três medidas. No entanto, ao comparar apenas as medidas de 3 e 5 minutos, a prevalência de $\mathrm{HO}$ aumenta de 2,0 para $2,6 \%$. Embora esses indivíduos tendam a ser mais sintomáticos aos 5 minutos, o aumento da sensibilidade para a triagem de 
HO é pequeno, e certamente não justifica estender as medidas além de 3 minutos durante uma avaliação no consultório.

Mas talvez um dos aspectos mais interessantes deste trabalho tenha sido o cálculo dos escores $Z$ para variações da PA, observando valores inferiores aos estabelecidos pelas diretrizes para um subconjunto específico da população. A distribuição da variação da PA resultou em escores Z -2 de $-14,09 \mathrm{mmHg}$ para PAS e $-5,39 \mathrm{mmHg}$ para PAD na subamostra de pacientes sem hipertensão, diabetes, história de insuficiência cardíaca, doença arterial coronariana, infarto do miocárdio prévio ou acidente vascular cerebral. Isso significa que, nesta coorte de adultos brasileiros, os atuais limiares nacionais e internacionais podem subestimar a presença de HO. Essa diferença pode, inclusive, explicar a menor prevalência nessa coorte brasileira. Como existem reflexos autonômicos envolvidos na resposta fisiológica da PA em pé, ${ }^{1}$ é razoável admitir que poderíamos ter variações diferentes para diferentes populações. Em outras palavras, um número não pode servir para todos. O estudo de Velten et al. ${ }^{10}$ fornece dados para uma discussão mais ampla sobre esse assunto. Obviamente, mais dados serão necessários em populações diversas, uma vez que o estudo ELSA-Brasil avaliou apenas uma amostra específica de funcionários de seis instituições brasileiras.

Independentemente se entraremos em discussões sobre os limiares de HO no país - se uma queda de 20 ou $14 \mathrm{mmHg}$ na PAS - , isso não muda o fato de que o problema pode continuar sendo negligenciado na prática clínica. Existe uma recomendação formal para medir a PA no 1o e $3^{\text {o }}$ minutos após ficar em pé, partindo de uma posição sentada, em todos os pacientes na primeira avaliação no consultório para identificar a HO. ${ }^{2-4}$ As medidas da PA em repouso e em pé também devem ser consideradas em visitas subsequentes em pacientes idosos, diabéticos e pessoas com outras condições em que a $\mathrm{HO}$ pode ocorrer com frequência. No entanto, mesmo sabendo das suas possíveis implicações na incidência de eventos cardiovasculares, a $\mathrm{HO}$ é frequentemente subdiagnosticada e pode ser um problema negligenciado na prática clínica.

Aproximadamente dois terços dos pacientes com $\mathrm{HO}$ não serão detectados se as medidas sequenciais da PA na posição vertical não forem realizadas na prática. ${ }^{13}$ Mesmo em um estudo clínico desenvolvido para avaliar a eficácia da monitorização ambulatorial da PA na detecção da $\mathrm{HO}$, apenas $76 \%$ dos 505 pacientes foram triados durante visitas regulares ao consultório. ${ }^{14} \mathrm{~A}$ falta de tempo durante as consultas pode ser um dos principais fatores. Além disso, agora pode-se argumentar que a prevalência de $\mathrm{HO}$ em indivíduos de meia-idade é realmente baixa, questionando se devemos realizar uma triagem sistemática conforme recomendado. No entanto, não há dúvidas sobre as implicações prognósticas da $\mathrm{HO}$, principalmente em idosos. Talvez essa discussão sobre hipotensão postural mereça a devida atenção para melhorar nossa sensibilidade, identificando quem realmente precisa ser avaliado e quais seriam as variações esperadas da PA para cada grupo de indivíduos.

\section{Referências}

1. Ricci F, De Caterina R, Fedorowski A. Orthostatic Hypotension: Epidemiology, Prognosis, and Treatment. J Am Coll Cardiol. 2015;66(7):848-60.

2. Malachias MVB, Souza WKSB, Plavnik FL, Rodrigues CIS, Brandão AA, Neves MFT, et al., Sociedade Brasileira de Cardiologia. 7a Diretriz Brasileira de Hipertensão Arterial. Arq Bras Cardiol. 2016;107(Supl.3):1-83.

3. Whelton PK, Carey RM, Aronow WS, Casey DE Jr, Collins KJ, Dennison Himmelfarb C, et al. 2017ACC/AHA/AAPA/ABC/ACPM/AGS/APhA/ASH/ ASPC/NMA/PCNA Guideline for the Prevention, Detection, Evaluation, and Management of High Blood Pressure in Adults: Executive Summary: A Report of the American College of Cardiology/American Heart Association Task Force on Clinical Practice Guidelines. Hypertension. 2018;71(6):1269-324.

4. Williams B, Mancia G, Spiering W,Agabiti Rosel E, Azizi H, Burnier H, et al. 2018 ESC/ESH Guidelines for the management of arterial hypertension. Eur Heart J. 2018;39(33):3021-104.

5. Kaufmann H. Consensus statement on the definition of orthostatic hypotension, pure autonomic failure and multiple system atrophy. Clin Auton Res. 1996; 6(2):125-6.

6. Verwoert GC, Mattace-Raso FU, Hofman A, Heeringa J, Stricker BH, Breteler MM, et al. Orthostatic hypotension and risk of cardiovascular disease in elderly people: the Rotterdam study. J Am Geriatr Soc. 2008;56(10):1816-20.

7. Eigenbrodt ML, Rose KM, Couper DJ, Arnett DK, Smith R, Jones D. Orthostatic hypotension as a risk factor for stroke: the Atherosclerosis Risk in Communities (ARIC) study, 1987-1996. Stroke. 2000;31(10):2307-13

8. Ricci F, Fedorowski A, Radico F, Romanello M, Tatasciore A, Di Nicola $M$, et al. Cardiovascular morbidity and mortality related to orthostatic hypotension: a meta-analysis of prospective observational studies. Eur Heart J. 2015;36(25):1609-17.

9. Fagard RH, De Cort P. Orthostatic hypotension is a more robust predictor of cardiovascular events than nighttime reverse dipping in elderly. Hypertension. 2010;56(1):56-61.

10. Velten APC, Bensenor I, Lotufo P, Mill JG. Prevalence of Orthostatic Hypotension and the Distribution of Pressure Variation in the Longitudinal Study of Adult Health. Arq Bras Cardiol. 2020; 114(6):1040-1048.

11. Schmidt MI, Duncan BB, Mill JG, Lotufo PA, Chor D, Barreto M, et al. Cohort profile: longitudinal study of adult health (ELSA-Brasil). Int J Epidemiol. 2015;44(1):68-75

12. Low PA. Prevalence of orthostatic hypotension. Clin Auton Res. 2008;18(Suppl 1):8-13.

13. Carlson JE. Assessment of orthostatic blood pressure: measurement technique and clinical applications. South Med J. 1999;92(2):167-73.

14. Cremer A, Rousseau AL, Boulestreau R, Kuntz S, Tzourio C, Gosse P, et al. Screening for orthostatic hypotension using home blood pressure measurements. J Hypertens. 2019 May;37(5):923-27. 